\title{
Prediction of building interference effects on pedestrian level comfort
}

\author{
A.D. Ferreira ${ }^{\mathrm{a}, *}$, A.C.M. Sousa ${ }^{\mathrm{b}}$, D.X. Viegas ${ }^{\mathrm{a}}$ \\ ${ }^{a}$ Department of Mechanical Engineering, University of Coimbra, Polo II, 3030-290 Coimbra, Portugal \\ ${ }^{\mathrm{b}}$ Department of Mechanical Engineering, University of New Brunswick, P.O. Box 4400, Fredericton, NB, \\ Canada E3B $5 A 3$
}

\begin{abstract}
This paper presents results concerning the interference effect created by two auxiliary buildings located upstream of a recreation area comprising seven pavilions. Both numerical and experimental simulations were used to conduct the analyses, and the experimental and numerical results are compared against each other for vertical velocity profiles at different locations. For the numerical simulation, the RANS equations were solved with the turbulence formulated by the $k-\varepsilon R N G$ model. For the experimental work, a $\frac{1}{175}$ scale model was tested in a low-speed wind tunnel. (C) 2002 Elsevier Science Ltd. All rights reserved.
\end{abstract}

Keywords: Pedestrian comfort; Building interference; Numerical simulation; Turbulence

\section{Introduction}

The modification of wind pattern in a built-up area due to adjacent buildings can be quite significant. Interference effects depend on several conditions and, in particular, on the upstream obstacles and terrain conditions, geometry and arrangement of neighbouring structures, and on their orientation with respect to the wind direction. Depending on the level of aerodynamic interference, its effects may significantly affect the efficiency of building ventilation systems [1,2], wind loads [3], air quality [4,5], pedestrian comfort [6], just to mention a few.

For the analysis and evaluation of interference effects, two main approaches have been used: wind tunnel and computational fluid dynamics (CFD). CFD, as discussed

\footnotetext{
*Corresponding author. Tel.: +351-239-790-727; fax: +351-239-790-771.

E-mail address: almerindo.ferreira@dem.uc.pt (A.D. Ferreira).
} 
by Gosman [7], has been gaining widespread acceptance by taking advantage of the tremendous progress of computer capabilities in recent years, and also from advances in numerical modelling. In fact, to assess the environmental impact of planned urban developments, modelling is preferred over field measurements, as these are costly and not suitable for optimisation studies, as discussed in detail by Plate [5]. In spite of the capabilities of experimental modelling, where a scale model is needed and tested in a wind tunnel, the technique is sometimes characterised by high time requirements and high costs, especially if optimisation studies are to be undertaken and detailed measurements are needed. It should be mentioned, however, that often, when only minor changes in the physical model's configuration and/or geometry are required for a parametric evaluation, data acquisition via wind tunnel may prove to be highly expeditious and reliable at moderate costs.

In the recent past, several studies have focussed on the wind-induced interference effects. Kandhuri et al. [8] used a neural network approach to establish some generalisation rules, keeping in mind design loads. However, as mentioned by the same authors in Ref. [9], the few attempts at providing general recommendations have not been successful due to the complex nature of the problem and the large discrepancies among the various studies. In fact, each situation is, to a great extent, unique and therefore specific studies are required for different physical arrangements or conditions, as shown, for instance, in the computational approach performed by He and Song [10].

The present work aims to analyse the interference generated by two auxiliary structures upon the wind velocity and wind pattern in a recreation area, where a high level of pedestrian comfort is required. The computational approach was the prime analysis tool; however, experimental simulation was performed to evaluate the performance of the numerical model in this complicated configuration. The experimental simulation was performed just for one wind-incidence direction, with and without the auxiliary buildings. For a more complete analysis of the wind flow in the recreation area, the prevailing site wind directions were numerically simulated [11], and local measurements were performed [12].

\section{Case study}

The area under study is located on the north bank of Tagus river, within the space where the '98 World Exposition (Lisbon, Portugal) took place. The present study is addressed to the South International Area (SIA), a zone of the site where seven pavilions, separated by passageways, were located.

Fig. 1 depicts the top and east side views of the area studied, with indication of actual dimensions. The top view also indicates, with dashed lines, the overhangs of the pavilions' roofs that partly cover the passageways. Due to the large difference between the horizontal and vertical dimensions, the east view was drawn with a vertical scale twice as large as the horizontal scale. Buildings R1 and R2 represent two auxiliary areas (information centres and restaurants) and, as requested by the 


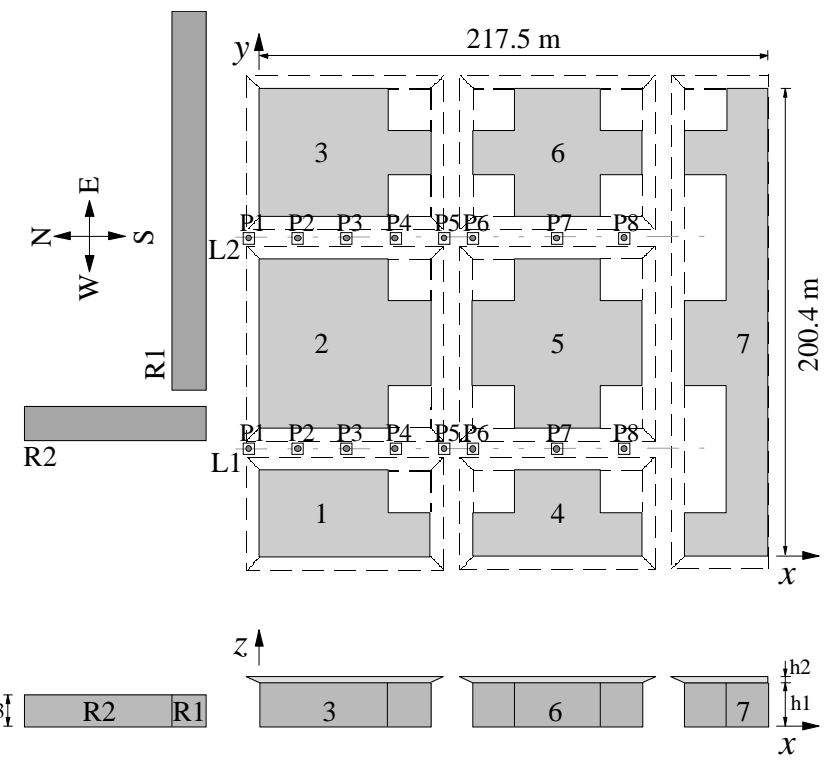

Fig. 1. Top- and east-side views of the area under study. The dimensions correspond to the full size $(\mathrm{h} 1=9.1 \mathrm{~m} ; \mathrm{h} 2=1.4 \mathrm{~m} ; \mathrm{h} 3=6.5 \mathrm{~m})$ (the vertical scale is twice the horizontal scale).

EXPO organisation, the influence upon interference of wind speed and pattern within the pavilions' area was analysed.

Wind records performed in the area over several years indicate that the prevailing wind directions are from North-Northwest. Taking into account the location of R1 and R2 structures relative to the pavilions' area, the strongest interference effects are expected to occur for wind directions blowing from the North quadrant. In this way, R1 and R2 are located upstream of the pavilions' area, therefore only three directions were considered for the interference study. Several other directions, ranging from Northwest to East, were numerically simulated, with R1 and R2 in place $[11,12]$, corresponding to the final architectural configuration chosen for the EXPO site.

For the experimental simulation, a $\frac{1}{175}$ scale model was tested in a large low-speed, open wind tunnel installed at the Association for the Development of Industrial Aerodynamics (ADAI, Coimbra, Portugal) laboratory. Several vertical profiles were measured using both a single hot-film probe as well a seven-hole probe, taking advantage of its capabilities to perform measurements in situations where the wind flow is not aligned with the probe [13]. The top view of Fig. 1 shows the different locations (P), along the passageways L1 and L2, where the vertical profiles were measured. The interference data acquisition was carried out just for the NorthSouth wind direction, as it corresponds to the alignment for which the strongest influence of R1 and R2 is expected. Moreover, the measuring devices preclude to a great extent reliable measurements for different wind directions. 


\section{Numerical model}

For the numerical simulation, it is assumed that the wind flow through the passageways is governed by the three-dimensional, incompressible, Reynoldsaveraged, steady-state equations of conservation of mass and momentum. These equations can be formulated as follows:

$$
\begin{aligned}
& \frac{\partial u_{i}}{\partial x_{i}}=0 \\
& \frac{\partial}{\partial x_{j}}\left(\rho u_{j} u_{i}\right)=-\frac{\partial p}{\partial x_{i}}+\frac{\partial}{\partial x_{j}}\left[\mu\left(\frac{\partial u_{i}}{\partial x_{j}}+\frac{\partial u_{j}}{\partial x_{i}}\right)-\rho \overline{u_{i}^{\prime} u_{j}^{\prime}}\right] .
\end{aligned}
$$

The standard formulation of the $k-\varepsilon$ model from Launder and Spalding [14] was adopted as the base form for the evaluation of the Reynolds stress tensor, $-\rho \overline{u_{i}^{\prime} u_{j}^{\prime}}$. Using the eddy viscosity concept, where $\mu_{t}=\mathrm{C}_{\mu} \rho k^{2} / \varepsilon$, this model is expressed by the following equations:

$$
\begin{aligned}
& -\rho \overline{u_{i}^{\prime} u_{j}^{\prime}}=\mu_{t}\left(\frac{\partial u_{i}}{\partial x_{j}}+\frac{\partial u_{j}}{\partial x_{i}}\right)-\frac{2}{3} \delta_{i j} \rho k \\
& \frac{\partial}{\partial x_{j}}\left(\rho u_{j} k\right)=\frac{\partial}{\partial x_{j}}\left[\left(\mu+\frac{\mu_{t}}{\sigma_{k}}\right) \frac{\partial k}{\partial x_{j}}\right]+\mu_{t}\left(\frac{\partial u_{i}}{\partial x_{j}}+\frac{\partial u_{j}}{\partial x_{i}}\right) \frac{\partial u_{i}}{\partial x_{j}}-\rho \varepsilon \\
& \frac{\partial}{\partial x_{j}}\left(\rho u_{j} \varepsilon\right)=\frac{\partial}{\partial x_{j}}\left[\left(\mu+\frac{\mu_{t}}{\sigma_{\varepsilon}}\right) \frac{\partial \varepsilon}{\partial x_{j}}\right]+C_{\varepsilon 1} \frac{\varepsilon}{k} \mu_{t}\left(\frac{\partial u_{i}}{\partial x_{j}}+\frac{\partial u_{j}}{\partial x_{i}}\right) \frac{\partial u_{i}}{\partial x_{j}}-C_{\varepsilon 2} \rho \frac{\varepsilon^{2}}{k} .
\end{aligned}
$$

In the previous equations, $u_{i}$ stands for the mean velocity component in the $x_{i}$ direction (tensor notation), $p$ the mean pressure, $\rho$ the density, $\varepsilon$ the dissipation rate of turbulence energy, $k$ the turbulent energy given by $k=\overline{u_{i}^{\prime} u_{i}^{\prime}} / 2, \mu$ and $\mu_{t}$ the molecular and eddy viscosity, respectively, $\sigma_{k}, \sigma_{\varepsilon}, c_{\varepsilon 1}, c_{\varepsilon 2}$ and $c_{\mu}$ being the turbulence model constants.

For the turbulence modelling, the RNG extension of the $k-\varepsilon$ model [15] was adopted, as this model provides an improvement, when compared with the $k-\varepsilon$ model, in flows where recirculation zones occur, as found by different authors $[11,16,17]$. These results, to some extent, contradict the negative assessment of the model made by Nagano and Itazu [18].

Using the renormalisation group theory, Yakhot et al. [15] determined all the model constants, and suggested the inclusion of an extra term on the right-hand side of the dissipation transport of the $k-\varepsilon$ standard model (Eq. (5)). The extra term $R$ depends on the rate of strain, and is formulated as

$$
R=-\frac{\rho C_{\mu} \eta^{3}\left(1-\eta / \eta_{0}\right)}{1+\beta \eta^{3}} \frac{\varepsilon^{2}}{k},
$$

where $\eta=S k / \varepsilon$ and $S^{2}=2 S_{i j} S_{i j}, S_{i j}$ being the mean strain tensor. The different constants were set according to Table 1 , where the $k-\varepsilon$ standard model constants are also indicated for comparison purposes. 
Table 1

Turbulence model constant values

\begin{tabular}{llllllll}
\hline Model & $C_{\mu}$ & $C_{\varepsilon 1}$ & $C_{\varepsilon 2}$ & $\sigma_{k}$ & $\sigma_{\varepsilon}$ & $\eta_{0}$ & $\beta$ \\
\hline$k-\varepsilon$ & 0.09 & 1.45 & 1.9 & 1.0 & 1.3 & - & - \\
RNG & 0.085 & 1.42 & 1.68 & 0.72 & 0.72 & 4.38 & 0.015 \\
\hline
\end{tabular}

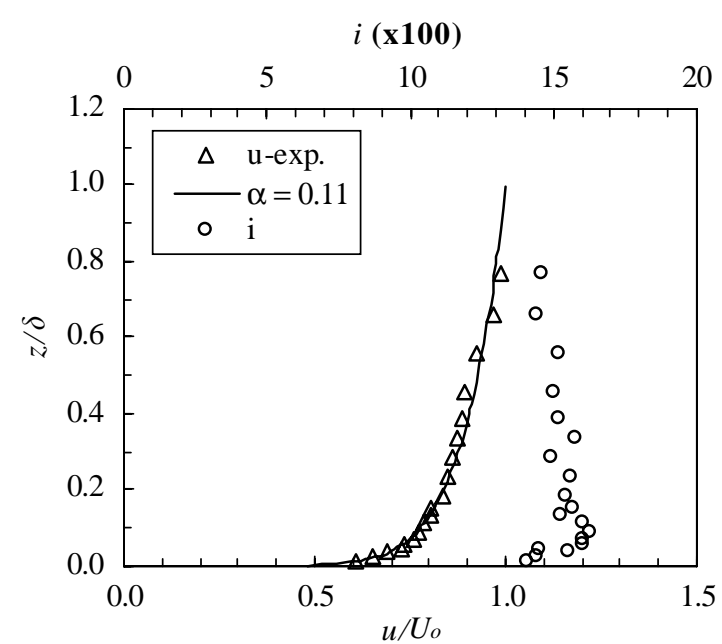

Fig. 2. Inlet conditions for the velocity and turbulence intensity (results measured in the wind tunnel).

The control volume formulation was chosen for the discretisation of the transport equations in a Cartesian coordinate system. Continuity and momentum equations are linked through the pressure correction in accordance with the SIMPLEC formulation [19].

The experimental values measured in the wind tunnel for the streamwise velocity component $(u)$ were used for the inlet boundary conditions of the velocity and the turbulent kinetic energy $(k)$, this last one calculated as a function of the turbulence intensity $(i)$ from the equation $k=3 / 2(u i)^{2}$. The inlet velocity profile can be approximated by a power law:

$$
\frac{u}{U_{0}}=\left(\frac{z}{\delta}\right)^{\alpha}
$$

for which the exponent $\alpha$ was found to be around 0.11 , which corresponds quite well to the conditions of flow over a nearly flat terrain or a large surface of water (Fig. 2). In the experiments, the undisturbed velocity value $U_{0}$, measured outside the boundary layer, was set to be $=11 \mathrm{~m} / \mathrm{s}$, and a boundary layer thickness $(\delta)$ of $0.4 \mathrm{~m}$ was observed $0.5 \mathrm{~m}$ upstream of the model's pavilions 1 and 3 . Full details on the boundary conditions and numerical procedures can be found in Ref. [12]. 


\section{Results}

In this section, some experimental and computational results are presented, depicting the influence of structures R1 and R2 (Fig. 1) upon the velocity field in all the pavilions' area, taking into consideration only winds from Northwest (NW), North (N) and Northeast (NE) directions. For evaluation of the numerical model performance, several measurements were conducted, namely horizontal velocity profiles [11] as well as vertical ones in different locations labelled P1-P8 along two passageways L1 and L2, according to Fig. 1. As mentioned before, the vertical profiles were measured only for the North-wind direction.

Figs. 3 and 4 show, respectively, the evolution of the velocity magnitude $(V)$ variation relative to the inlet flow conditions at the same level $\left(V_{0}\right)$, at different vertical profiles along the $\mathrm{L} 1$ corridor, in the absence of R1 and R2 and when those auxiliary structures are in place. The modulus of the velocity difference is normalised by the undisturbed velocity $U_{0}$, measured above the boundary layer thickness $(\delta)$ :

$$
\frac{\left(V-V_{0}\right)}{U_{0}}
$$

The relation between R1's height (equal to R2's) and the boundary-layer thickness is about 0.1 .

As can be noticed, the numerical predictions agree fairly well with the experimental results. The major discrepancies in Fig. 3 are observed at the lower levels for the first position P1, which can be explained based on a small ground step simulated in the experimental model but not reproduced in the numerical model. Predictions, as shown in Figs. 3 and 4, with and without the R1 and R2 structures, respectively, indicate that the vertical velocity difference modulus is affected by the presence of the structures, particularly for P2 and P3 locations at the L1 corridor

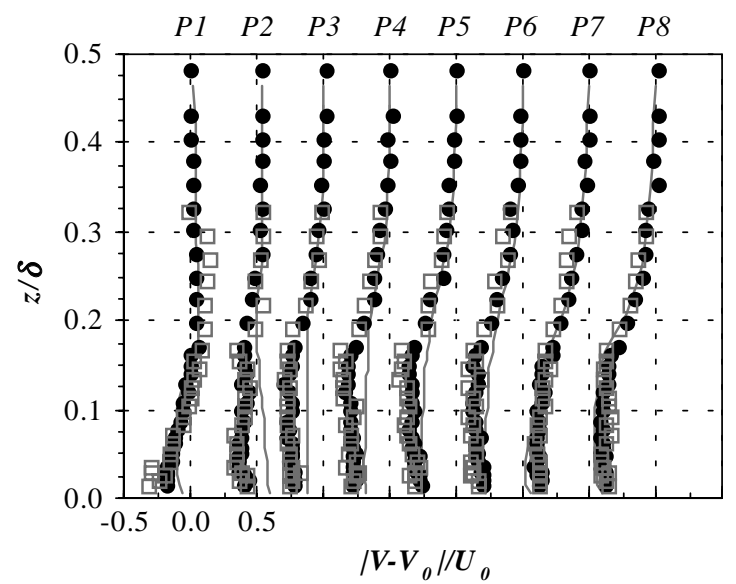

Fig. 3. Velocity profiles along passage L1 in the absence of R1 and R2-North-wind direction (line: numerical results; circles: hot-wire measurements; squares: seven-hole probe measurements). 


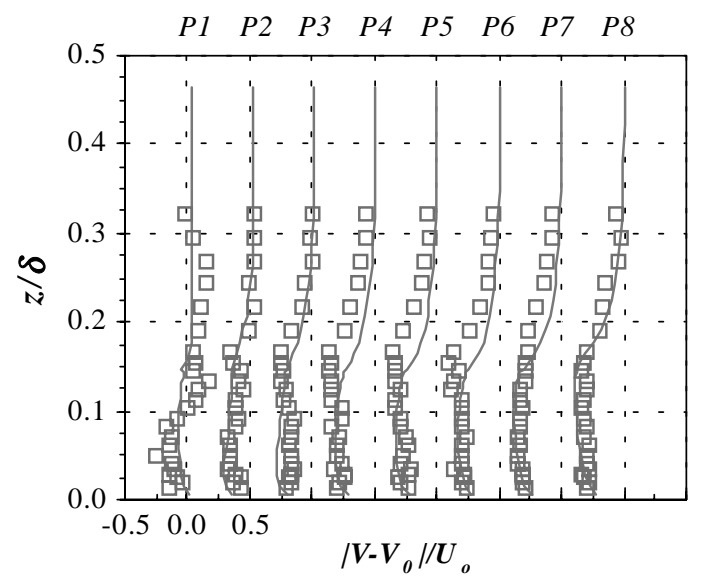

Fig. 4. Velocity profiles along passage L1 when R1 and R2 are present-North-wind direction (line: numerical results; squares: seven-hole probe measurements).

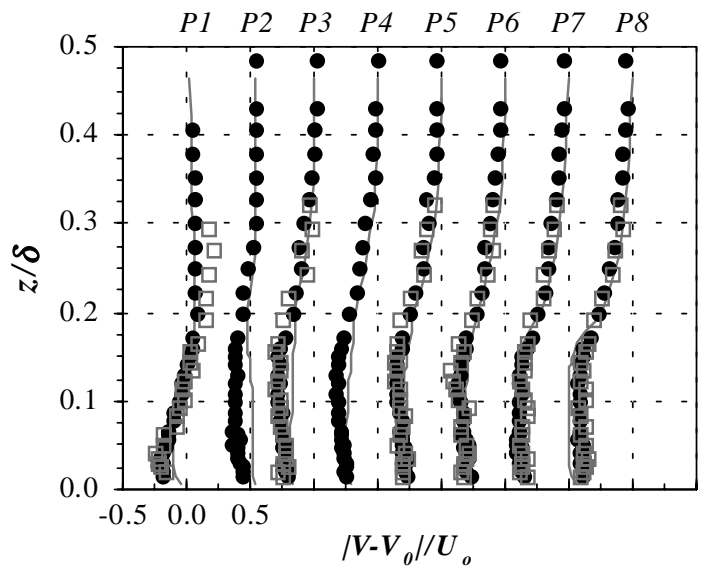

Fig. 5. Velocity profiles along passage L2 in the absence of R1 and R2-North-wind direction (line: numerical results; circles: hot-wire measurements; squares: seven-hole probe measurements).

entrance area. This finding is not fully corroborated in those locations by the measurements (Figs. 3 and 4). Altogether Figs. 3 and 4, under close observation, establish that the presence of the structures has a negligible influence upon the velocity modulus along L1 corridor, at best, and primarily based on the numerical results, it yields a minor decrease in the velocity modulus. The overall agreement between the experimental and computational results is good, in particular, for the L1 downstream locations.

Figs. 5 and 6 show a similar set of results but for the L2 corridor, with and without the R1 and R2 structures present, respectively. For the first case, the major 


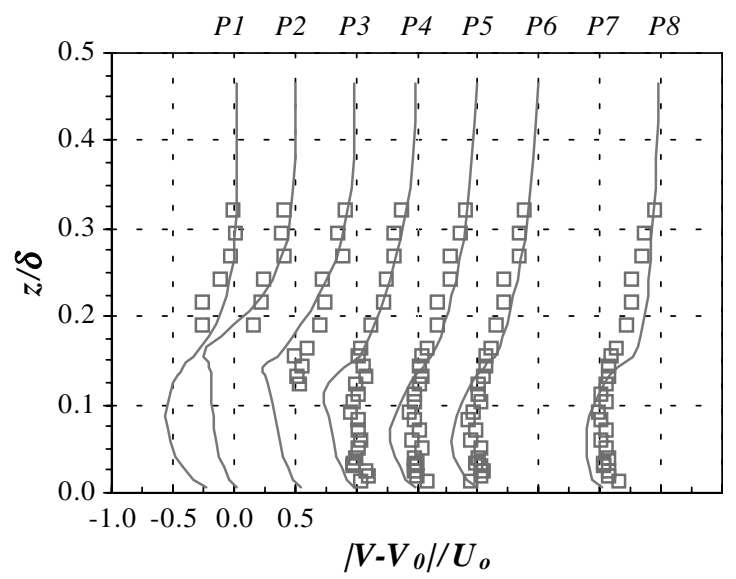

Fig. 6. Velocity profiles along passage L2 when R1 and R2 are present-North-wind direction (line: numerical results; squares: seven-hole probe measurements).

discrepancy can be observed in the lower part of the $\mathrm{P} 2$ profile, an observation similar to that made for corridor L1. The agreement, however, is good for all other locations.

Analysing now the profiles along L2, when R1 and R2 are considered, some major discrepancies can be observed, especially for the upstream positions. The essence of the flow around R1 is somewhat similar to the problem of a surface-mounted obstacle, or even to the classical problem of the flow over a backward-facing step. Other authors have observed similar predictions of shortcomings even when using more complex models, as mentioned, e.g. in Refs. [20-23].

In Fig. 6, for the profiles P1 and P2, due to measuring device limitations, it was not possible to obtain information for the lower levels, as the flow in that region showed strong vorticity and flow reversal relative to the probe's alignment. This fact, taking into account the good agreement between experimental and numerical results, emphasises some of the advantages of the numerical model upon the experimental simulation.

The topology of the flow, numerically predicted, is depicted in Figs. 7 and 8 at the mean vertical plane along the L2 corridor, with and without the R1 and R2 buildings, respectively, and reveals a long recirculation zone downstream of the R1 structure.

As a result, and as expected, the vorticity generated by the presence of R1 induces a strong velocity modulus reduction in its wake region as compared to the configuration when both R1 and R2 structures are not in place.

This effect is clearly evident in Fig. 9 where the velocity field, at a $3 \mathrm{~m}$ high level, obtained when R1 and R2 are absent, is normalised by the correspondent field when the structures are present. A maximum velocity ratio value of $\approx 12$ was predicted at the entrance region of L2 corridor, showing the significant interference effect induced by the R1 building. 


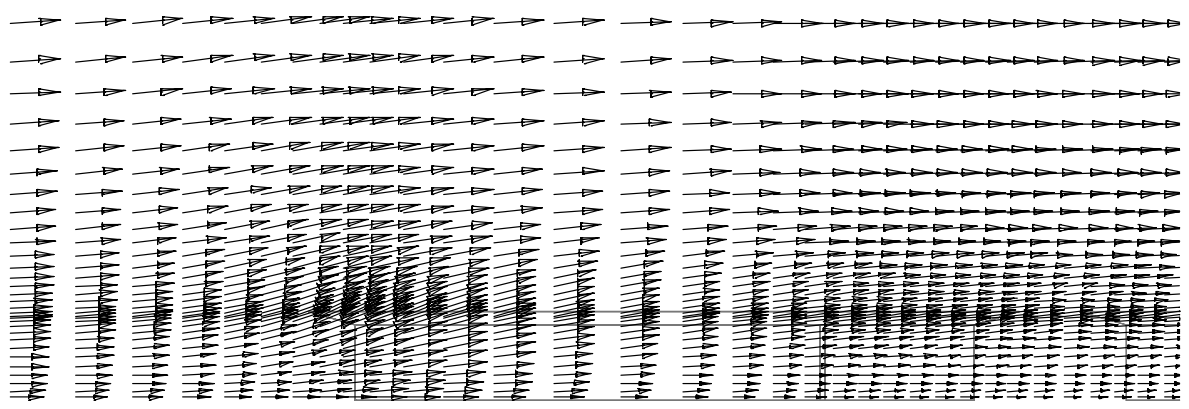

Fig. 7. Numerical velocity field predicted for the L 2 vertical plane in the absence of R1 and R2 structures.

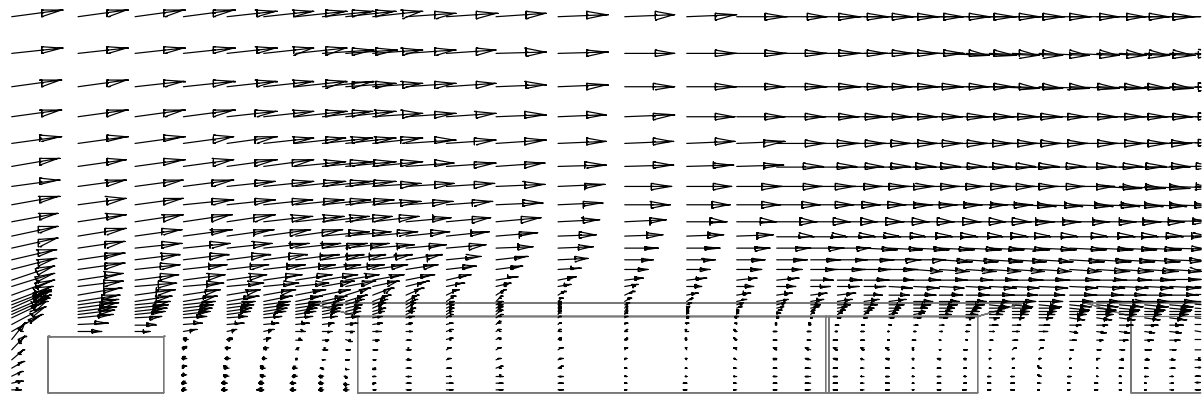

Fig. 8. Numerical velocity field predicted for the L2 vertical plane when R1 and R2 structures are present.

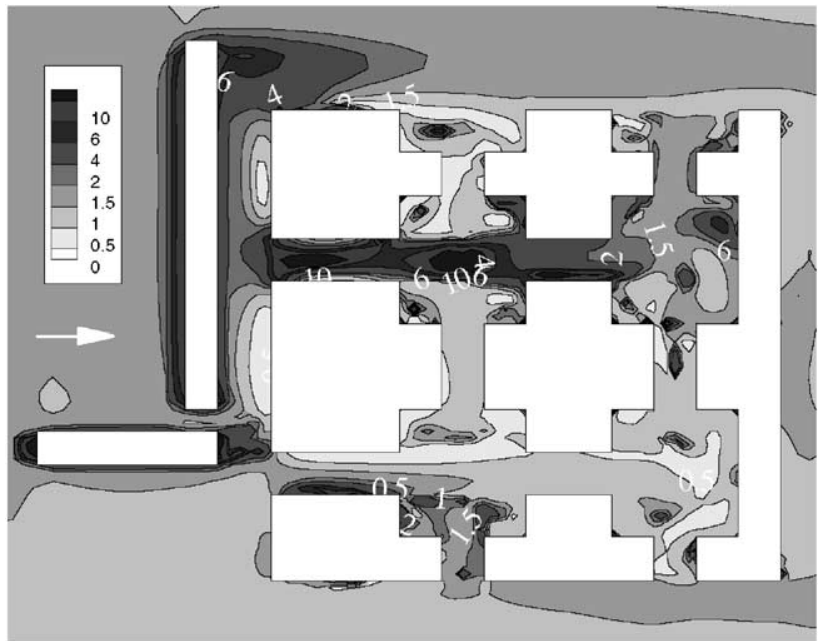

Fig. 9. Velocity field, at $z=3 \mathrm{~m}$ and for North-wind direction, calculated without R1 and R2 and normalised by the correspondent field with R1 and R2. 


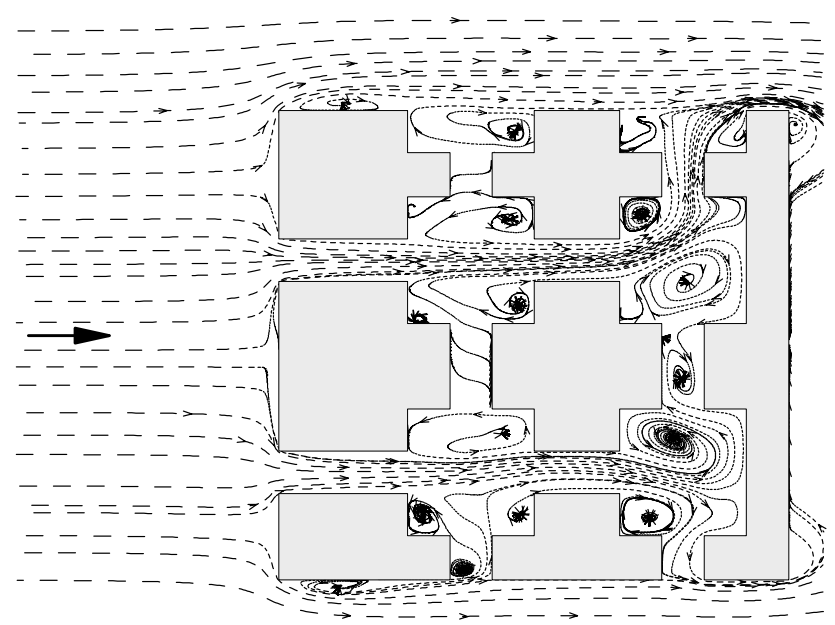

Fig. 10. Streamlines at $z=3 \mathrm{~m}$, for North-wind direction, in the absence of R1 and R2 structures.

Integrating the velocity field over all the pavilions' area, the computational simulation predicts a general wind-speed reduction of $\approx 40 \%$ due to the presence of R1 and R2 auxiliary structures.

In addition to reduced velocity zones, some acceleration areas can also be noticed, namely at the north and west walls of pavilion 2. Structure R2 channels are a part of the incoming flow into the L1 passageway, however the acceleration effect is only noticeable close to pavilion 2 . The model also predicts a significant reduction of velocity in the passageway adjacent to pavilion 1 when $\mathrm{R} 1$ and $\mathrm{R} 2$ are present. This effect can be explained based on the flow deflection induced at the west far end of the upwind wall of pavilion 1, combined with the shelter effect resulting from the south end of building R2, as illustrated in Figs. 10 and 11 where the streamlines, at $z=3 \mathrm{~m}$ level and for North-wind direction, are depicted for the cases without and with the R1 and R2 structures present, respectively.

Taking into account the R1 and R2 locations, the influence of the structures is expected to be negligible upon the flow field in the pavilions' area, for winds blowing from the South quadrants. Therefore only the cases for the Northwest and Northeast-wind directions are to discussed.

For the Northwest-wind direction, the streamline patterns, presented in Figs. 12 and 13, reveal a flow deceleration at the entrance zone of L2 corridor induced by the presence of R1 and R2. This fact is clearly identified when the velocity modulus ratio is represented, as shown in Fig. 14. From the figure, it can be said that R1 and R2 create a channelling effect, inducing stronger velocities near the north wall of pavilion 2, where an increase of $50 \%$ is predicted as compared to the situation where $\mathrm{R} 1$ and $\mathrm{R} 2$ are not in place. In general, in the pavilions' area, the presence of R1 and R2 produce an approximate reduction of $25 \%$ on the velocity intensity, for Northwest-wind direction. 


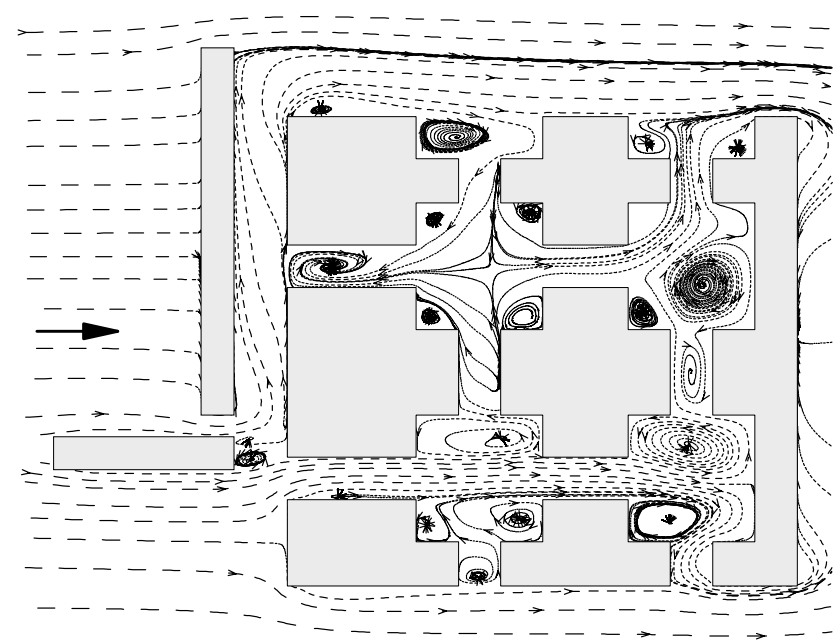

Fig. 11. Streamlines at $z=3 \mathrm{~m}$, for North-wind direction, when R1 and R2 structures are present.

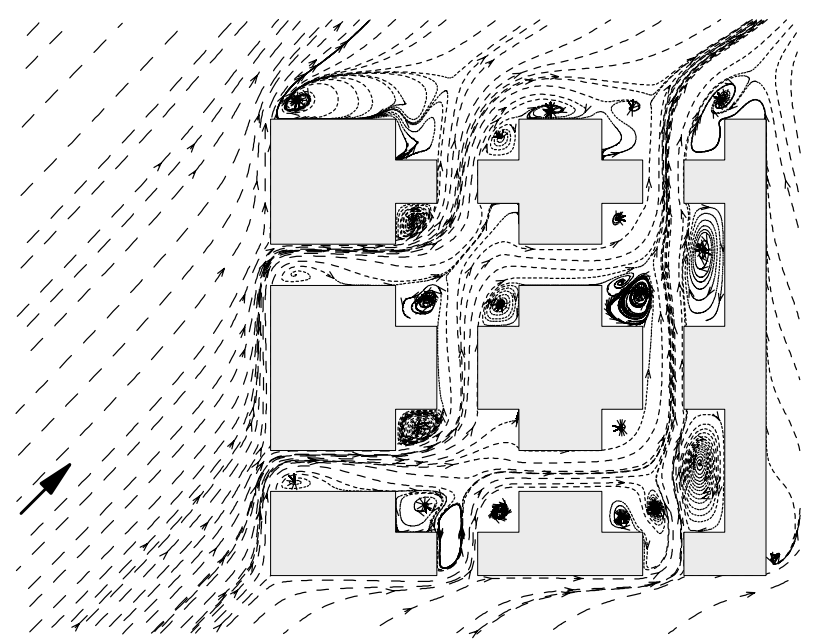

Fig. 12. Streamlines at $z=3 \mathrm{~m}$, for Northwest-wind direction, in the absence of R1 and R2 structures.

For the Northeast-wind direction, a stronger influence is predicted, as shown in Figs. 15-17. It can be noticed that R1 and R2 produce a strong shelter effect upon the corridors L1 and L2, reducing the velocity intensity up to $\frac{1}{6}$ of what it would be without those structures. In fact, the flow pattern changes considerably from one situation to the other, especially in the narrow passages along corridors L1 and L2 as depicted in Figs. 15 and 16. By integrating the velocity ratio over all the pavilions' involving area, a reduction of $45 \%$ is predicted by the numerical model, so a clear improvement for the pedestrian comfort is achieved by the presence of R1 and R2 


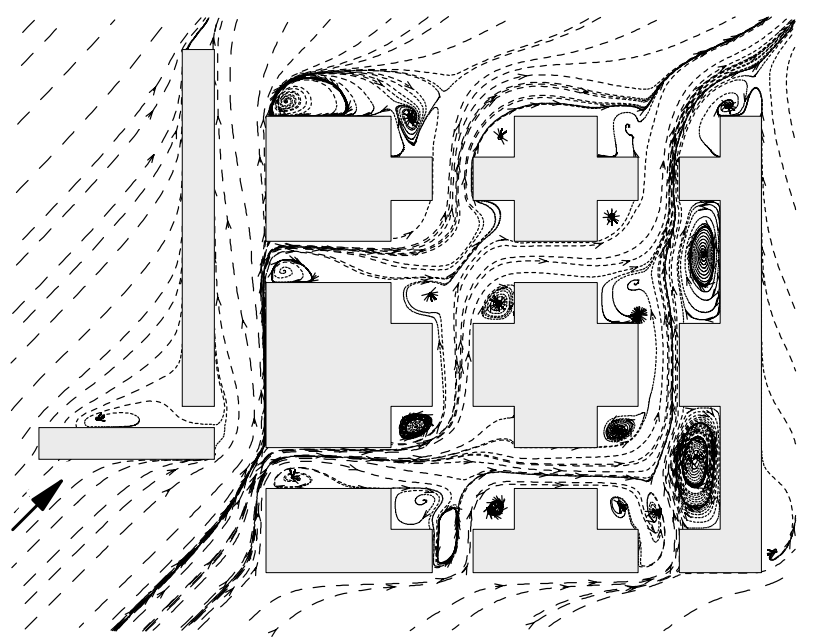

Fig. 13. Streamlines at $z=3 \mathrm{~m}$, for Northwest-wind direction, when R1 and R2 structures are present.

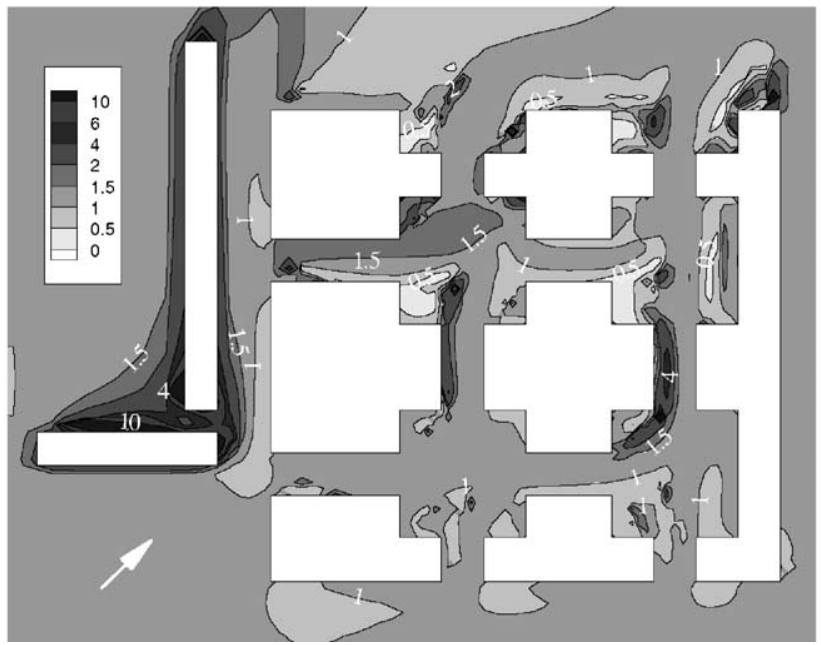

Fig. 14. Velocity field, at $z=3 \mathrm{~m}$ and for Northwest-wind direction, calculated without R1 and R2 and normalised by the correspondent field with R1 and R2.

structures, considering this comfort purely in terms of wind intensity, since the flow was taken as isothermal.

\section{Conclusions}

A numerical and experimental study was presented concerning the evaluation of the interference and shelter effect generated by the presence of two structures upon a 


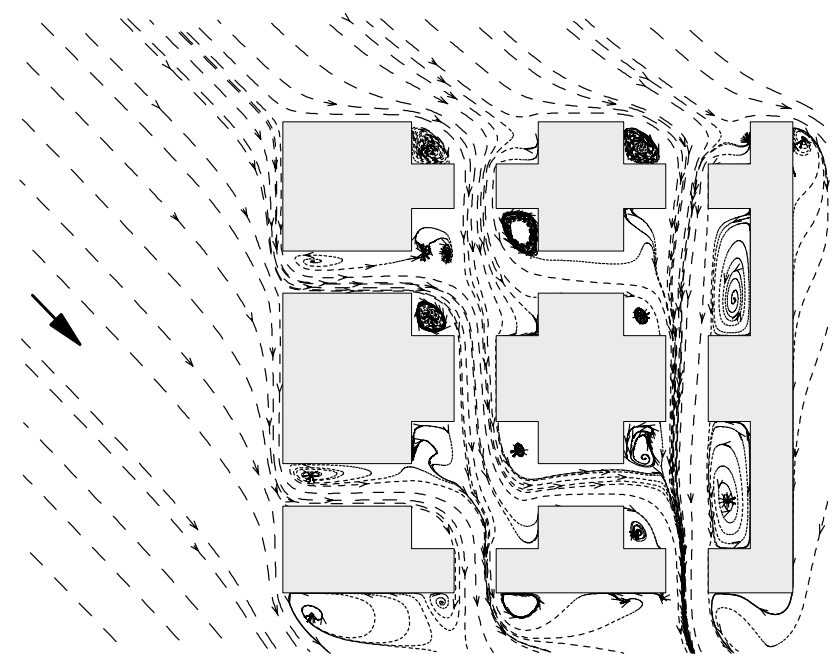

Fig. 15. Streamlines at $z=3 \mathrm{~m}$, for Northeast-wind direction, in the absence of R1 and R2 structures.

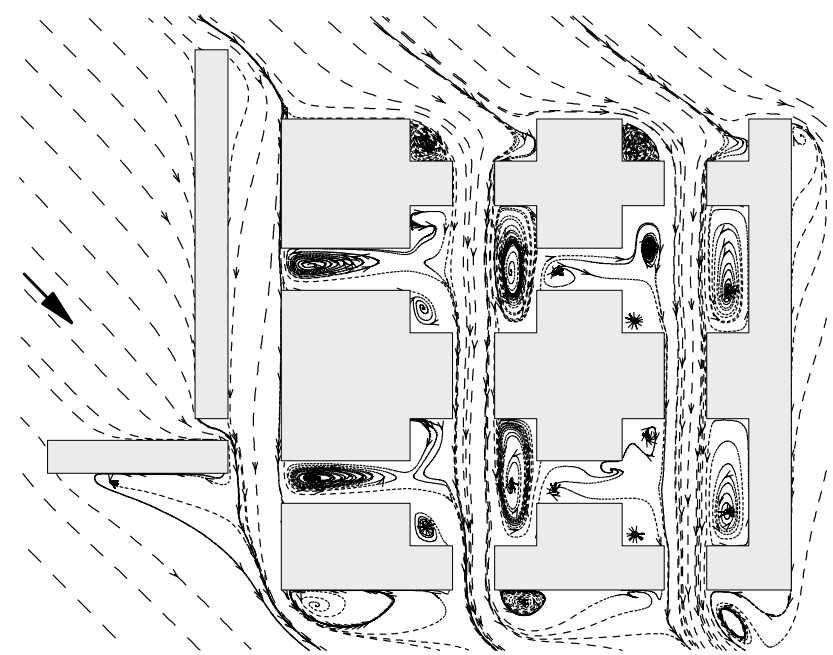

Fig. 16. Streamlines at $z=3 \mathrm{~m}$, for Northeast-wind direction, when $\mathrm{R} 1$ and $\mathrm{R} 2$ structures are present.

recreation area where several pavilions, separated by passageways, are located. The comparison between the numerical and experimental sets of velocity results, for different vertical profiles, with and without the two auxiliary structures, reveal good agreement. This gives further evidence towards the credibility and potential advantages of a well-tuned numerical model, as it provides relatively fast and reliable information for the overall area under consideration.

It was found that the presence of the two buildings increases the pedestrian level of comfort, as it reduces the velocity magnitude at the entrance of one of the corridors 


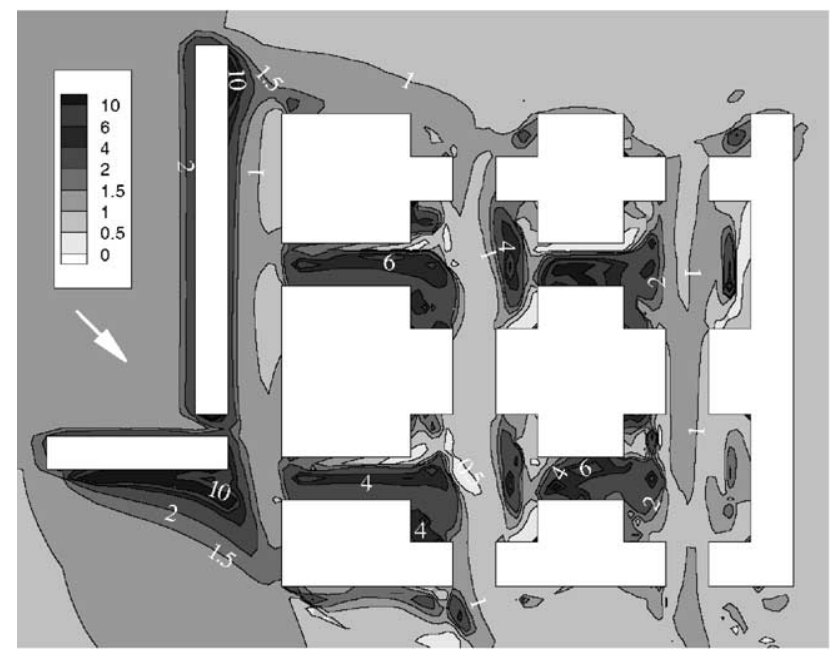

Fig. 17. Velocity field, at $z=3 \mathrm{~m}$ and for Northeast-wind direction, calculated without R1 and R2 and normalised by the correspondent field with R1 and R2.

to a minimum of $\approx \frac{1}{10}$ of its value when the structures are not present. In some specific locations of the L1 passageway, however, increases of around $100 \%$ were also noted.

The numerical model predicts an overall wind-speed reduction, due to the presence of the two auxiliary structures, ranging from $25-45 \%$, for wind directions from Northwest to Northeast. As a consequence, it can be said that the presence of the structures significantly contributes to the improvement of the pedestrian level comfort.

\section{References}

[1] R. Jozwiak, J. Kacprzyk, J.A. Zuranski, Wind tunnel investigations of interference effects on pressure distribution on a building, J. Wind Eng. Ind. Aerodyn. 57 (1995) 159-166.

[2] R. Jozwiak, J. Kacprzyk, J.A. Zuranski, Influence of wind direction on natural ventilation of apartment buildings, J. Wind Eng. Ind. Aerodyn. 60 (1996) 167-176.

[3] H.-J. Niemann, H.-D. Köpper, Influence of adjacent buildings on wind effects on cooling towers, Eng. Struct. 20 (1998) 874-880.

[4] M. Bottema, Towards rules of thumb for wind comfort and air quality, Atmos. Environ. 33 (1999) 4009-4017.

[5] E.J. Plate, Methods of investigating urban fields - physical models, Atmos. Environ. 33 (1999) 3981-3989.

[6] M. Bottema, A method for optimisation of wind discomfort criteria, Build. Environ. 35 (2000) 1-18.

[7] A.D. Gosman, Developments in CFD for industrial and environmental applications in wind engineering, J. Wind Eng. Ind. Aerodyn. 81 (1999) 21-39.

[8] A.C. Khanduri, T. Stathopoulos, C. Bédard, Modelling wind-induced interference effects using backpropagation neural networks, J. Wind Eng. Ind. Aerodyn. 72 (1997) 71-79. 
[9] A.C. Khanduri, T. Stathopoulos, C. Bédard, Wind-induced interference effects on buildingsa review of the state-of-the-art, Eng. Struct. 20 (1998) 617-630.

[10] J. He, C.C.S. Song, Evaluation of pedestrian winds in urban area by numerical approach, J. Wind Eng. Ind. Aerodyn. 81 (1999) 295-309.

[11] A.D. Ferreira, A.C.M. Sousa, D.X. Viegas, Numerical and experimental simulation of the wind field in the EXPO'98 area, Wind Struct. 1 (1998) 337-349.

[12] A.D. Ferreira, A.C.M. Sousa, D.X. Viegas, Numerical and experimental study of the wind flow around a group of low-rise pavilions, Proceedings of the 10th International Conference on Wind Engineering, Copenhagen, Denmark, 21-24 June 1999, pp. 1271-1276.

[13] M.C.G. Silva, D.X. Viegas, Calibration and use of a non-nulling seven-hole pressure probe, AGARD CP-535-Wall Interference, Support Interference and Flow Field Measurements, 1994, pp. 1-7.

[14] B.E. Launder, D.B. Spalding, The numerical computation of turbulent flows, Comp. Meth. Appl. Mech. Eng. 3 (1974) 269-289.

[15] V. Yakhot, S.A. Orszag, S. Thangam, T.B. Gatski, C.G. Speziale, Development of turbulence models for shear flows by a double expansion technique, Phys. Fluids A 4 (1992) 1510-1520.

[16] L.M. Smith, S.L. Woodruff, Renormalization-group analysis of turbulence, Annu. Rev. Fluid Mech. 30 (1998) 275-310.

[17] G.C. Papageorgakis, A. Arbor, Comparison of linear and non-linear RNG-based $k-\varepsilon$ models for incompressible turbulent flows, Numer. Heat Transfer, Part B 1 (1999) 1-22.

[18] Y. Nagano, Y. Itazu, Renormalization group theory for turbulence: assessment of the YakhotOrszag-Smith theory, Fluid Dyn. Res. 20 (1997) 157-172.

[19] J.P. Van Doormaal, G.D. Raithby, Enhancements of the SIMPLE method for predicting incompressible fluid flows, Numer. Heat Transfer 7 (1984) 147-163.

[20] R.S. Amano, P. Goel, Investigation of third-order closure model of turbulence for the computation of incompressible flow in a channel with a backward-facing step, J. Fluids Eng. 109 (1987) 424-428.

[21] S. Acharya, S. Dutta, T.A. Myrum, R.S. Baker, Turbulent flow past a surface-mounted twodimensional rib, J. Fluids Eng. 116 (1994) 238-246.

[22] F.S. Lien, M.A. Leschziner, Assessment of turbulence-transport models including non-linear RNG eddy-viscosity formulation and second-moment closure for flow over a backward-facing step, Comput. Fluids 23 (1994) 983-1004.

[23] R.R. Hwang, Y.C. Chow, Y.F. Peng, Numerical study of turbulent flow over two-dimensional surface-mounted ribs in a channel, Int. J. Numer. Methods Fluids 31 (1999) 767-785. 